\title{
Achalasia: un trastorno de la motilidad esofágica, no tan raro
}

\author{
Ma Pilar Orgaz Gallego
}

\begin{abstract}
a Médico de Familia.
CEDT Tarancón (Cuenca).

Correspondencia:

$\mathrm{M}^{\mathrm{a}}$ Pilar Orgaz Gallego,

Centro de Salud de Tarancón,

$\mathrm{C} /$ Camino Corral de Almaguer s/n, 16400-Tarancón (Cuenca).

E-mail: mporgaz@sescam.jccm.es.
\end{abstract}

Recibido el 18 de septiembre de 2008

Aceptado para su publicación el 10 de noviembre de 2008

\begin{abstract}
RESUMEN
La Achalasia es un raro trastorno de la motilidad esofágica distal, de etiología desconocida. La destrucción irreversible de las neuronas del plexo mientérico esofágico, causa aperistalsis y fallo en la relajación del esfínter esofágico inferior durante la deglución. Consecuentemente, el bolo alimenticio se detiene a nivel del cardias, produciendo disfagia y otros síntomas subsecuentes como regurgitación, dolor torácico, pirosis y pérdida de peso. Una historia clínica cuidadosa, la endoscopia digestiva alta o el estudio esófagogastroduodenal con papilla de bario pueden sospecharla y ser confirmada mediante manometría esofágica. Otras enfermedades, especialmente malignas, pueden imitar los síntomas clínicos. La dilatación neumática con balón es la terapia de primera elección en la acalasia, aunque la miotomía de Heller está emergiendo en muchos centros como tratamiento de elección, porque ha reducido significativamente la invasividad y el coste del procedimiento. Especialmente en pacientes jóvenes, debe ofertarse esta técnica junto a funduplicatura como procedimiento antirreflujo, cuando la terapia dilatadora falla. Presento dos casos de achalasia, mostrando que, aunque la incidencia es aproximadamente de 1 por 100.000 personas/año, no es tan infrecuente en la práctica clínica como pudiéramos pensar.
\end{abstract}

Palabras clave. Acalasia. Trastornos Motores Esofágicos. Disfagia.

\section{ABSTRACT}

Achalasia: a not so rare esophageal motility disorder

Achalasia is a rare motility disorder of the distal esophagus, of unknown etiology. The irreversible destruction of esophageal myenteric plexus neurons causes aperistalsis and the failure of lower esophageal sphincter to relax during deglutition. Consequently, the food bolus stops at the level of the cardia producing disphagia and other symptoms such as regurgitation, chest pain, heartburn and weight loss. With a thorough medical history, upper Gl endoscopy and barium swallow X-ray a diagnosis of achalasia can be suspected and later confirmed by esophageal manometry. Other diseases, especially malignant diseases, can imitate the clinical symptoms. Pneumatic balloon dilatation is the first choice treatment for achalasia, but Heller myotomy is now becoming the treatment of choice in many hospitals because it is significantly less invasive and costly. Heller myotomy together with laparoscopic fundoplication in order to prevent reflux should be offered when dilatation therapy fails, especially in younger patients. I present two cases of achalasia, demonstrating that, although the incidence is approximately 1 per 100,000 people per year, it is not so infrequent in clinical practice as one would think.

Key words. Achalasia. Deglutition Disorders. Dysphagia.

\section{INTRODUCCIÓN}

La disfagia, ó sensación de detención del bolo alimenticio durante la deglución, es el síntoma fundamental de los trastornos motores esofágicos (TME), suele ser intermitente y no progresiva, aumentando su prevalencia con la edad. Se clasifica en orofaríngea y esofágica. Una detallada historia clínica aportará los datos suficientes para llegar a su diagnóstico hasta en el $80 \%$ de los $\operatorname{casos}^{1,2}$. La achalasia, a pesar de su infrecuencia, es la alteración motora del esófago más habitual ${ }^{2}$.

\section{OBSERVACIONES CLÍNICAS}

Se presentan dos casos de "achalasia ó acalasia". El primero, un varón de 40 años que consultó por disfagia de un año de evolución, sin odinofagia, regurgitación, vómitos, 
ni pirosis. En tratamiento desde hacía 3 semanas con Domperidona 10mg/8h, sin mejoría. En una consulta posterior refirió que con la ingestión de alimentos sólidos notaba la detención del bolo alimenticio, lo que le obligaba a beber agua para que progresase el alimento. No presentaba astenia, anorexia ni pérdida de peso. No tenía antecedentes de hernia hiatal, ulcus, ni ingesta previa de caústicos. Con esta clínica se derivó a Digestivo diagnosticándole acalasia. El estudio baritado (figura 1), muestra un esófago dilatado de forma difusa por encima del esfínter esofágico inferior (EEI) y afilamiento de la luz esofágica distal. A los 15 días fue intervenido quirúrgicamente por laparoscopia "Miotomía de Heller junto a Funduplicatura anterior", con evolución clínica satisfactoria (figura 2).

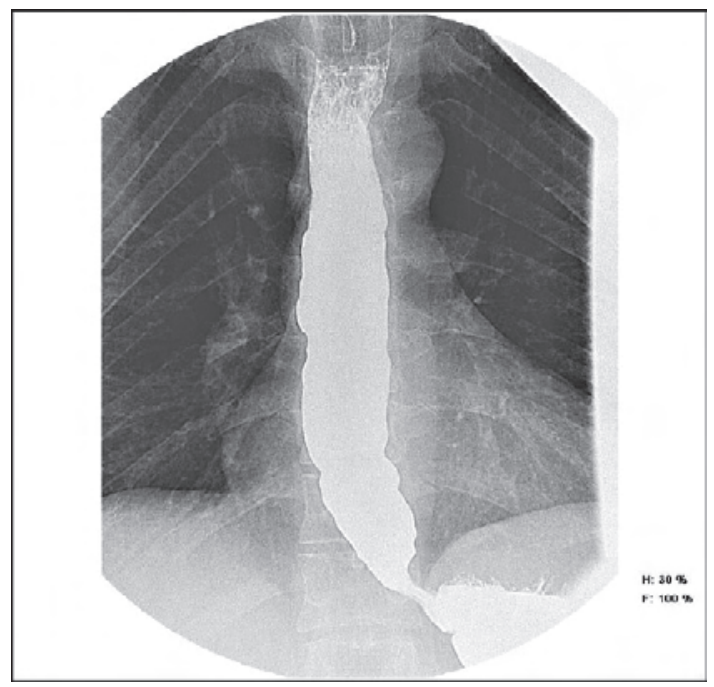

Figura 1. Caso 1: estudio baritado en el que se muestra un esófago dilatado de forma difusa por encima del esfínter esofágico inferior y afilamiento de la luz esofágica distal.

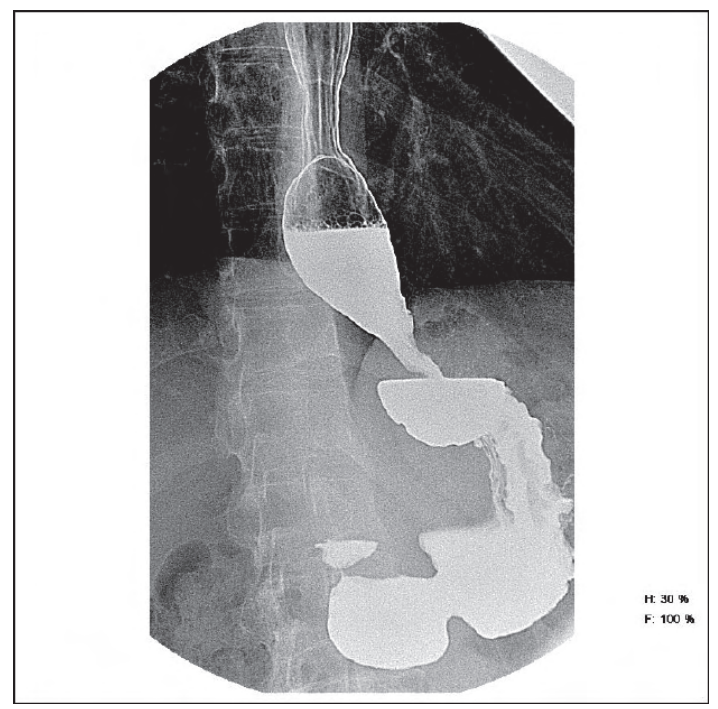

Figura 2. Caso 1: Miotomía de Heller junto a Funduplicatura anterior", con evolución clínica satisfactoria.

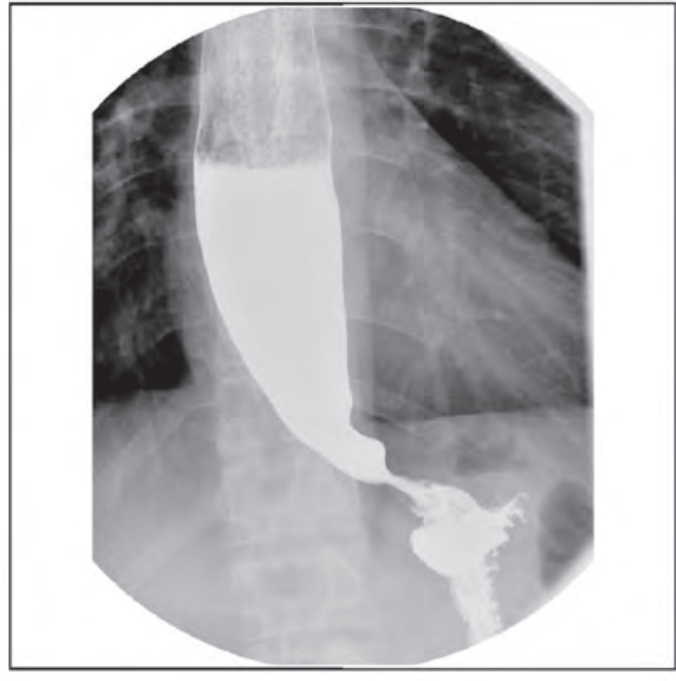

Figura 3. Caso 2: discreta estenosis esofágica distal con dilatación proximal.

El segundo caso es otro varón de 33 años de edad sin hábitos tóxicos conocidos, que consultó por disfagia esofágica distal a sólidos, desde hacía unos meses. Relataba textualmente la sensación de tener una bola en epigastrio que no le dejaba el paso a los alimentos sólidos, en ocasiones asociado a pirosis. No nauseas ni vómitos. A veces épocas de estreñimiento. Comía muy deprisa, de todo, y no había perdido el apetito. Tras tratamiento con Domperidona y Omeprazol, notó discreta mejoría. Se solicitó EEGD con papilla de bario desde atención primaria (figura 3), informado como "discreta estenosis esofágica distal con dilatación proximal, siendo aconsejable descartar achalasia, no objetivando reflujo gastroesofágico". Fue remitido a consulta de Digestivo, realizándole pruebas complementarias: hemograma, estudio de hierro, bioquímica general y hepática, función tiroidea, proteinograma, pruebas reumáticas y estudio autoinmune, rigurosamente normales, detectándose un déficit parcial de factor VIII de la coagulación, con homocisteína en rango alto y anticuerpos antifosfolípidos negativos. EI TAC torácico con contraste informó de discreta dilatación esofágica en toda su longitud hasta la unión esófagogástrica, con engrosamiento parietal, sin objetivarse masas, muy sugestivo de achalasia. Parénquima pulmonar y pleura sin hallazgos. Durante la realización de todas estas pruebas complementarias, siguió tratamiento con Cinitaprida $1 \mathrm{mg} / 8 \mathrm{~h}$, en espera de tratamiento quirúrgico.

\section{COMENTARIOS}

Acalasia (en griego "ausencia de relajación") es el trastorno motor esofágico (TME) mejor conocido de todos, aunque poco frecuente. Ya descrita en 1672, 
como "cardioespasmo", su etiología aún es desconocida, al menos en la forma esporádica, más habitual en el mundo noroccidental. Afecta a ambos sexos por igual con una incidencia de 1-2 casos por 100.000 individuos/año $0^{2,3}$. Se caracteriza por el fracaso en la relajación del EEl durante la deglución y la aperistalsis del cuerpo del esófago, lo que conduce a la dilatación de éste (megaesófago) por encima del EEl y al estasis alimentario, responsables de la clínica ${ }^{4}$.

La sintomatología característica es la disfagia, tanto para sólidos como para líquidos: el paciente experimenta sensación de parada del bolo, que suele localizar con precisión, aunque no siempre, en el cardias. Otras veces refiere la necesidad de beber para permitir el paso del bolo al estómago. La regurgitación de alimentos y saliva, especialmente por la noche, también es un síntoma frecuente, con el riesgo añadido de neumonía por aspiración y abscesos pulmonares. El dolor torácico, en ocasiones desencadenado por el acto de la deglución, se da con mayor frecuencia en fases iniciales de la enfermedad. En algunos casos refieren pirosis y, si la disfagia es franca, pueden llegar a desnutrirse y presentar una pérdida ponderal importante ${ }^{2,5}$.

Las investigaciones efectuadas en el último decenio han demostrado que la base de estas alteraciones motoras del cuerpo esofágico es la denervación selectiva (al menos en fases iniciales de la enfermedad) de las neuronas del plexo mientérico liberadoras de mediadores miorrelajantes (péptido intestinal vasoactivo, óxido nítrico) y la prevalencia relativa de neuronas que liberan sustancias como acetilcolina, que producen contracción. La causa de esa denervación se desconoce y se han propuesto mecanismos autoinmunes, infecciones (Tripanosoma Cruzi en la enfermedad de Chagas, herpes virus o Morbillivirus) e incluso genéticos (Síndrome triple AAA). Estudios recientes han mostrado en formas iniciales de la enfermedad la presencia de un infiltrado linfocitario en el plexo mientérico del EEI, lo que confirma la hipótesis inflamatoria en la patogenia de la acalasia ${ }^{5}$.

El diagnóstico se basa en la clínica y en las pruebas complementarias siguientes:

- Endoscopia: indicada en pacientes mayores de 45 años para descartar otras causas de disfagia (anillo de Schatzki y estenosis benignas ó malignas).Se puede apreciar la dilatación del esófago y el afilamiento distal de la luz esofágica, con cierta resistencia al paso de endoscopio hacia la cavidad gástrica, sin alteración orgánica.
- Radiografía baritada: muestra la típica imagen del esófago distal en "cola de ratón", " pico de pájaro" ó "punta de lápiz" y un esófago dilatado por encima del EEI con ausencia de peristalsis.

- Rx simple de tórax: se puede apreciar un nivel hidroaéreo por detrás del corazón.

- Manometría esofágica: estudio de elección ante la sospecha de acalasia cuando la radiografía es normal o no concluyente. Muestra ausencia de peristaltismo del cuerpo esofágico (criterio obligado), un aumento de la presión del EEl en reposo (aunque puede ser normal), relajación incompleta o inexistente del EEI (verdadero responsable de la obstrucción al paso del alimento al estómago) y una elevación de la presión del cuerpo esofágico (igual ó superior a la gástrica), así como ondas espontáneas (no precedidas de deglución) 4 .

- En algunos casos, y según la clínica, es recomendable realizar TAC, RMN o técnicas endoscópicas más complejas ${ }^{6}$.

Existen situaciones clínicas de otra etiología que presentan una sintomatología similar; es bien conocida la relación entre cáncer esofágico y acalasia de larga evolución. Sin embargo, procesos neoplásicos de cardias ó esófago distal pueden imitar clínicamente a la acalasia ${ }^{7}$. El diagnóstico diferencial entre acalasia y seudoacalasia puede ser difícil con los métodos de rutina y se debe sospechar ante edad avanzada, disfagia de rápida instauración y falta de respuesta al tratamiento convencional (dilataciones). El uso de nitrito de amilo durante la exploración radiológica posibilita discriminar entre las dos entidades, permitiendo la apertura de la unión esófagogástrica en la acalasia idiopática, mientras que no causa ningún efecto en la seudoacalasia ${ }^{7,8}$. La cirugía exploratoria puede ser necesaria para el diagnóstico definitivo ${ }^{8}$.

En cuanto al tratamiento, al desconocer la causa, es paliativo y tiene como objetivo reducir la presión basal del EEI para permitir el tránsito esofagogástrico, evitando la aparición de reflujo gastroesofágico. Disponemos de una amplia variedad de opciones terapeúticas, aunque ninguna completamente eficaz:

- Medidas farmacológicas: anticolinérgicos, teofilina, beta2-agonistas, sildenafilo, antagonistas del calcio (nifedipino y diltiazem) y dinitrato de isosorbide.

- Técnicas endoscópicas:

- "Dilataciones neumáticas" con balón Rigiflex (actualmente es la técnica no quirúrgica, de elección). Menor eficacia en jóvenes. Respuesta del $60-80 \%$ a los dos años. Tasa de complicaciones (perforación) del $33 \%$, pues requiere múltiples sesiones. 
- "Inyecciones de toxina botulínica" intraesfinteriana. Técnica muy segura con $60-85 \%$ de eficacia inicial, pero limitada en el tiempo, ya que recidiva a los 6-12 meses en el $50 \%$ de los pacientes, por lo que queda restringido su uso a pacientes de alto riesgo con baja expectativa de vida.

- "Cardiomiotomía": Técnica más efectiva, con buen resultado inmediato en el $80-90 \%$ de los casos y a largo plazo. Considerarla en pacientes sin riesgo quirúrgico. Tiene baja mortalidad y debe asociarse a técnica antirreflujo "Funduplicatura", puesto que en el $10 \%$ de los pacientes intervenidos aparece reflujo gastroesofágico como complicación más frecuente.

En la selección del tratamiento y aunque la técnica más coste-efectiva sea la dilatación neumática, habrá que considerar la edad del paciente, comorbilidad, supervivencia y técnicas disponibles, así como las preferencias del paciente ${ }^{2,6,9}$.

\section{BIBLIOGRAFÍA}

1. Disfagia. Guías clínicas. Disponible en: http://www.fisterra.com/fisterrae/guias.asp?idGuia=478. (Consultado el 17/03/2008).
2. Argüelles Arias F, García Montes JM, Herrerías Gutiérrez JM. Alteraciones motoras esofágicas. Acalasia. Espasmo esofágico. Disfagia orofaríngea. Medicine 2008; 10(1):20-7.

3. Fisichella M. Achalasia. Disponible en: http://www.emedicine.com/med/TOPIC16.HTM. (Consultado el 15/04/2008).

4. Gisbert JP, Losa C, Barreiro A, Pajares JM. Acalasia esofágica. Revisión de sus aspectos clínicos, diagnósticos y terapéuticos. Rev Clin Esp 2000; 200(8):424-31.

5. Zaninotto G, Costantini M, Rizetto C, Ancona E. Acalasia: estrategias terapéuticas. Cir Esp 2004; 75(3):117-22.

6. Acalasia. Guía clínica de la Society for Surgery of the Alimentary Tract. Disponible en: http://www.cirugest.com/htm/revisiones/cir11-06-02.htm. (Consultado el 19/08/2008).

7. Martínez C, Targarona EM, Sainza S, Cerdán G, Novell J, Trias M. Seudoacalasia: un diagnóstico a considerar en la evaluación de la disfagia. Gastroenterol Hepatol 2000; 23(1): 14-5. Disponible en: http://www.infodoctor.org/www/ acalasiaesofagica.htm. (Consultado el 19/08/2008).

8. Gockel I, Trinh TT, Mildenberger P, Oberholzer.K, Schmitt $\mathrm{T}$, Eckardt VF, Junginger T. Achalasia or pseudoachalasia? Problems of diagnostic and treatment decisions in two cases. Dtsch Med Wochenschr 2008; 133 (7):2904. Disponible en: http://www.ncbi.nlm.nih.gov/pubmed/ 18253919? ordinalpos=11\&itool=EntrezSystem. (Consultado el 17/03/2008).

9. Ruiz de León San Juan A. Alternativas terapeuticas en acalasia. Editorial. Rev Esp Enferm Dig 2003; 95(1):5-8. 